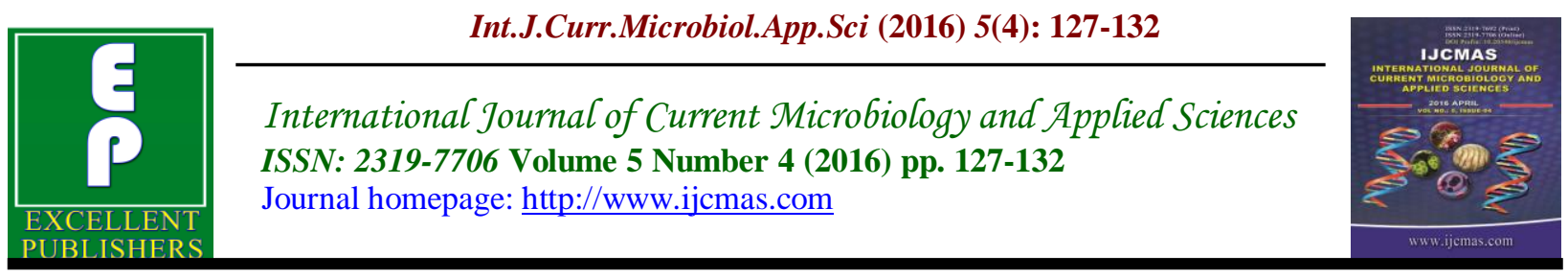

Original Research Article

http://dx.doi.org/10.20546/ijcmas.2016.504.017

\title{
Performance of Modified Hodge Test for Detection of Carbapenemase Producing Clinical Isolates of Pseudomonas aeruginosa
}

\author{
S. Jayalakshmi* and Sathya Pandurangan \\ Department of Microbiology, Shri Sathya Sai Medical College and Research Institute, Sri Balaji \\ Vidyapeeth University, Thiruporur, Kancheepuram (District), Tamilnadu, India \\ *Corresponding author
}

\begin{abstract}
A B S T R A C T
Keywords

Pseudomonas aeruginosa, Carbapenemase, Modified Hodge test (MHT), Beta-lactamase.

\section{Article Info}

Accepted:

09 March 2016

Available Online:

10 April 2016

Pseudomonas aeruginosa is a clinically troublesome gram negative pathogen that causes a wide range of opportunistic infections and nosocomial outbreaks. Carbapenems are becoming ineffective mostly due to the emergence of carbapenemase. The aim of this study was to performance of the phenotypic methods such as Modified Hodge test (MHT) for detection of carbapenemase producing clinical isolates of Pseudomonas aeruginosa. This study was conducted in the Department of Microbiology, SSSMC\&RI, which caters to 750 beds. A total of 152 Pseudomonas aeruginosa isolates from different clinical samples were taken, during the period of August 2015 to January 2016. These isolates were subjected to Modified Hodge Test. Out of 152 Pseudomonas aeruginosa isolates were tested for carbapenemase production by Modified Hodge test (MHT). Among the $152 P$. aeruginosa isolates, only 50(33\%) isolates were Modified Hodge test Positive and 102(67\%) isolates were negative by MHT. Modified Hodge test is a very sensitive and reliable test which can be performed in the routine laboratory test for detection of carbapenmase.
\end{abstract}

\section{Introduction}

Pseudomonas aeruginosa is a common Gram-negative bacilli associated with hospital infections and is often difficult to eradicate due to its resistant drug profile. However, carbapenems are being used as the last decade of the antimicrobial to treat serious infections due to MDR $P$. aeruginosa (Mahesh Kumar et al., 2016). After extended spectrum beta lactamases, another beta-lactamase causing resistance among Gram negative organisms is carbapenemase enzyme. This is an enzyme that hydrolyses a group of antibiotics called
Carbapenems (Gupta et al., 2006; Walsh et al., 2005). Carbapenemases belong to two major molecular families, are distinguished by the hydrolytic mechanism at the active site. The first carbapenemases described were the metalloenzymes as naturally. These enzymes utilised serine as their active sites (Nordman et al., 2011). Carbapenem resistance has been observed frequently in non-fermenting bacilli such as Pseudomonas aeruginosa and Acinetobacter spp. 
The common form of resistance is mediated by lack of drug penetration (i.e. porin mutation \& efflux pump) and carbapenem hydrolyzing beta-lactamases (Bansal et al., 2013). Mainly, three types of carbapenemases are now commonly identified in Enterobacteriaceae. They are the plasmid encoded IMP-1 (active on imepenem) a metallo- $\beta$-lactamase in $P$. aeruginosa, OXA-23, a class D Carbapenemase in Acinetobacter baumanii and KPC-1 a class A Carbapenemase in $K$. pneumonia (Ambler et al., 1991).

Unfortunately, resistance to carbapenems in Enterobacteriaceae is difficult to detect by routine disc diffusion method used by many microbiology laboratories (Sanjeev Kumar and Mehra, 2015). Various techniques can be used for detecting production of carbapenemase enzymes, like phenotypic to advanced molecular techniques. The Modified Hodge test (MHT) has been widely used as a general phenotypic method for the detection of carbapenemase production (Clinical and Laboratory Standard Institute, 2009).

The aim of this study was to performance of the phenotypic methods such as Modified Hodge test (MHT) for detection of carbapenemases producing clinical isolates of Pseudomonas aeruginosa.

\section{Materials and Methods}

\section{Bacterial Strains}

A prospective study was carried in a tertiary care hospital in south India over a period of six months (from August 2015 to January 2016) at the department of Microbiology. A total of 152 Pseudomonas aeruginosa were included in this study. These organisms were isolated from specimens like sputum, pus, High Vaginal swabs, Ear swab, Eye swab, Throat swab, DTT, stool, fluid, tissue,
COPD of patients admitted to different wards, which were sent to the Microbiology laboratory for routine culture, identification and sensitivity testing.

\section{Antimicrobial Susceptibility Testing and Phenotypic Screening}

The susceptibility test was carried out by Kirby-Bauer disc diffusion technique following the CLSI guidelines and interpretative criteria. Carbapenemase production was screened by disc diffusion; all the isolates with a reduced susceptibility to meropenem (diameter of zones of inhibition, $\leq 21 \mathrm{~mm}$ ) were considered as screening positive and further tested by phenotypic confirmatory methods.

\section{The Modified Hodge Test (MHT)}

In this test, a lawn culture of $E$. coli ATCC 25922 (1:10 dilution of 0.5 McFarland's standard broth) was done on a Mueller Hinton Agar (MHA) plate and allowed to dry for 2-5 minutes. A $10 \mu \mathrm{g}$ meropenem disk was placed in the centre of the plate. 10 $\mu l$ of $50 \mathrm{mM}$ zinc sulfate solution was added to meropenem disk. Further imepenem resistant $P$. aeruginosa isolate were taken, and then test organism was streaked in a straight line from the edge of the disc to the periphery of the plate. The plate was incubated overnight at $35 \pm 2^{\circ} \mathrm{C}$ in ambient air for 16-24 hours.

Quality control of the carbapenem discs were performed according to CLSI guidelines. Quality control of the following organisms MHT Positive Klebsiella pneumoniae ATCC BAA-1705 and MHT Negative Klebsiella pneumonia ATCC BAA-1706 (Centers for Disease Control and Prevention.).

After 16-24 hours of incubation, examine the plate for a 'clover-leaf' shape 
indentation at the intersection of the test organism and the E. coli ATCC 25922, within the zone of inhibition of the carbapenem susceptibility disc (Fig. 1).

MHT Positive test has a clover leaf-like indentation of the E. coli ATCC 25922 growing along the test organism growth streak within the disk diffusion zone.

MHT Negative test has no growth of the $E$. coli ATCC 25922 along the test organism growth streak within the disk diffusion.

\section{Results and Discussion}

Out of 152 isolates examined during the study period. Pseudomonas aeruginosa strains were infected with $\mathrm{n}=77(51 \%)$ were males and $\mathrm{n}=75(49 \%)$ females patients admitted in different wards (Table 1). Showing gender distribution of patients infected with $P$. aeruginosa.

The distribution of $P$. aeruginosa in clinical sample is summarized in (Table 2). Sputum samples were most common source $\mathrm{n}=85(56 \%)$, followed by pus swabs were $\mathrm{n}=35(23 \%)$, High vaginal swabs were most seen $\mathrm{n}=23(15 \%)$. Among other samples, $P$. aeruginosa was recovered from ear swabs $\mathrm{n}=2(1 \%)$ and followed by eye swab, throat swab, DTT, stool, fluid, tissue, COPD were $\mathrm{n}=1(0.7 \%)$.

All the isolates were tested for carbapenemase by Modified Hodge test (MHT) (Table 3). showing results, detection of carbapenemase production by Modified Hodge test from Pseudomonas aeruginosa isolates. Among the $152 P$. aeruginosa isolates, only 50(33\%) isolates were Modified Hodge test Positive and 102(67\%) isolates were negative by MHT.

$P$. aeruginosa is a well-known isolate in hospital settings and has been frequently associated with nosocomial outbreaks among susceptible patients. $P$. aeruginosa can cause pneumonias, Urinary tract infections and bacteremia's as well as cause high morbidity and mortality in patients with due to chronic infections that cause pulmonary damage and respiratory insufficiency (Breidenstein et al., 2011). Infections due to $P$. aeruginosa are difficult to eradicate because of their elevated intrinsic resistance as well as their capacity to acquire resistance to different antibiotics. Among the Pseudomonas species is the commonest causes for the production of carbapenemase. Carbapenemases are beta lactamases which have the ability to hydrolyze penicillins, cephalosporins, carbapenems and monobactams (Sowmya et al., 2015).

In this study, the most common specimens received were sputum (56\%) and pus swabs $(23 \%)$ followed by high vaginal swabs $(15 \%)$. Especially in medicine, ICU, chest medicine patients had primary lung disease or respiratory co-morbdity conditions. $P$. aeruginosa was found frequently to cause respiratory and suppurative skin infections. Other authors also had similar findings (Zavascki et al., 2006). In our study reveals that, $P$. aeruginosa infection was predominantly found among males $51 \%$ and another study showed $85.7 \%$ were most commonly seen in males infected with $P$. aeruginosa (Mara et al., 2006).

In this study the MHT detected carbapenemase production in 50 isolates were positive out of $152 P$. aeruginosa isolates. In 2013, Rouchelle tellis et al., have been reported MHT detected MBL production in 55 isolates were positive out of 100 carbapenem resistant NFGNB (Rouchelle Tellis et al., 2013). One of the meropenem resistant $P$. aeruginosa was positive for carbapenemase by Modified 
Hodge test, but negative for MBL and Amp $\mathrm{C} \beta$-lactamase. This may be because of the production of carbapenemase other than MBL, which is not dependent on zinc, ion for its action. Accordingly, apart from MBL, other classes of carbapenemases (class A or D) can also be responsible for meropenem resistance in $P$. aeruginosa (Queenan and Bush, 2007).

Table.1 Gender Distribution of Patients Infected with P. aeruginosa

\begin{tabular}{|c|c|c|}
\hline $\begin{array}{c}\text { Gender distribution } \\
\text { of patients }\end{array}$ & Total cases $(\mathbf{n = 1 5 2})$ & $\begin{array}{c}\text { Percentage } \\
(\boldsymbol{\%})\end{array}$ \\
\hline Male & 77 & $51 \%$ \\
\hline Female & 75 & $49 \%$ \\
\hline Total & $\mathbf{1 5 2}$ & $\mathbf{1 0 0 \%}$ \\
\hline
\end{tabular}

Table.2 Distribution of $P$. aeruginosa in Clinical Samples

\begin{tabular}{|c|c|c|}
\hline $\begin{array}{c}\text { Sources of } \boldsymbol{P} . \\
\text { aeruginosa isolates }\end{array}$ & $\begin{array}{c}\text { Number of isolates } \\
\text { (n=152) }\end{array}$ & $\begin{array}{c}\text { Percentage } \\
\text { \% }\end{array}$ \\
\hline Sputum & 85 & $56 \%$ \\
\hline Pus & 35 & $23 \%$ \\
\hline HVS & 23 & $15 \%$ \\
\hline Ear swab & 2 & $1 \%$ \\
\hline Eye swab & 1 & $0.7 \%$ \\
\hline Throat swab & 1 & $0.7 \%$ \\
\hline DTT & 1 & $0.7 \%$ \\
\hline Stool & 1 & $0.7 \%$ \\
\hline Fluid & 1 & $0.7 \%$ \\
\hline Tissue & 1 & $0.7 \%$ \\
\hline COPD & 1 & $0.7 \%$ \\
\hline
\end{tabular}

Fig.1 Detection of Carbapenemase Production by Modified Hodge Test

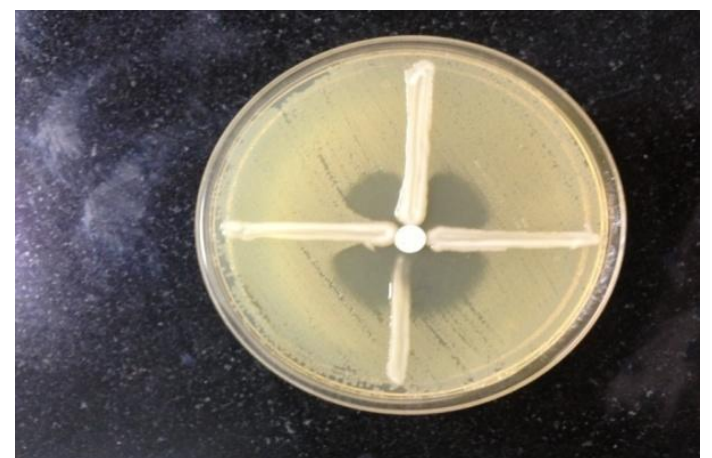

Modified Hodge test showing - Positive clover leaf pattern 
Table.3 Results of Modified Hodge Test from $P$. aeruginosa Isolates

\begin{tabular}{|c|c|}
\hline $\begin{array}{c}\text { Total no of } \boldsymbol{P} \text {. aeruginosa } \\
\text { isolates }\end{array}$ & $\begin{array}{c}\text { Detection of carbapenemase } \\
\text { production By Modified Hodge test }\end{array}$ \\
\hline$P$. aeruginosa isolates & $\mathrm{n}=152$ \\
\hline MHT Positive & $\mathrm{n}=50(33 \%)$ \\
\hline MHT Negative & $\mathrm{n}=102(67 \%)$ \\
\hline
\end{tabular}

The Modified Hodge test as it is described as a modified version of Hodge test, which was used some years ago. Even though the original Hodge test was evaluated utilizing the PCR confirmed IMP-1 and VIM-2 metallo $\beta$-lactamase (MBL) producing isolates. The original Hodge test utilized imipenem $10 \mu \mathrm{g}$ disc which gave fairly good results as it detected $67 \%$ cases of MBL producing Pseudomonas aeruginosa and Acinetobacter species. These isolates were evaluated by Modified Hodge test shown $100 \%$ sensitivity and specificity (Lee et al., 2003).

In another study was carried out in 2007 in Greece to evaluate different laboratory tests for detection of MBLs in Enterobacteriacae by Modified Hodge test was detected $98 \%$ cases from PCR as the gold standard, where as $0.03 \%$ was detected as false positive (Amjad et al., 2011). The drawbacks in our study are the small sample size and to evaluate the clinical isolates of Pseudomonas aeruginosa for detection of carbapenemase production by the Modified Hodge test only and not the comparison of the efficacy of different techniques for detection of carbapenemases. And further most studies are needed to evaluate the clinical usefulness of detection of carbapenemase.

This shows that Modified Hodge test is a very sensitive and reliable test for detection of carbapenemases. This necessity to need for detecting the resistant bacteria. So that unnecessary use of broad spectrum antimicrobials can be avoided. The finding of our study was having $50 \%$ of our isolates which showed intermediate or susceptible zone sizes on disc diffusion were detected positive by MHT which indicates the importance of this screening test.

In conclusion, Modified Hodge test is an easy and simple test to be performed to detect carbapenemase producing bacteria. There is a high percentage of carbapenemase producing Gram negative bacilli as a nonfermentors when compared to the different techniques for the detection of carbapenemase production by Modified Hodge test to avoid treatment failures and development of drug resistance due to unnecessary use of this class of antibiotic.

\section{Acknowledgement}

We would like to express gratitude to our management for the helpfulness.

\section{References}

Ambler, R.P., et al. 1991. A standard numbering scheme for the class A Blactamases. Biochem. J., 276: 269-272.

Amjad, A., Mirza, I.A., Abbasi, S.A., et al. 2011. Modified Hodge test: A simple and effective test for detection of carbapenemase production. Iran J. Microbiol., 3(4): 189-193.

Bansal, M., Vyas, N., Sharma, B., et al. 2013. Differentiation of carbapenemase producing Enterobacteriacae by triple disc test. Indian J. Basic Appl. Med. Res., 3: 314-320. 
Breidenstein, E.B.M., De la Fuente-Nufiez, C., Hancock, R.E.W. 2011. Pseudomonas aeruginosa: all roads lead to resistance. Trends Microbiol., 19: 419-426.

Centers for Disease Control and Prevention. Modified Hodge test for Carbapenemase detection in Enterobacteriacae. Available at http://www.ndhealth.gov/microlab/uplo ads/ Hodge Test.pdf.

Clinical and Laboratory Standard Institute. 2009 performance standards for antimicrobial susceptibility testing. Wayne, PA: Clinical and Laboratory Standards Institute, 2009.

Gupta, E., Mohanty, S., Sood, S., Dhawan, B., Das, B.K., Kapil, A. 2006. Emerging resistance to carbapenems in a tertiary care hospital in north India. Indian J. Med. Res., 124: 95-98.

Lee, K., Lim, Y.S., Yong, D., Yum, J.H., et al. 2003. Evaluation of the Hodge test and the imipenem- EDTA double disk synergy test for differentiating metallobeta-lactamase- producing isolates of Pseudomonas spp and Acinetobacter spp. J. Clin. Microbiol., 41: 4623-4629.

Mahesh Kumar, K., Chithralekha Saikumar. 2016. Detection of Metallo- $\beta$-lactamase production in Pseudomonas aeruginosa by Various Phenotypic Methods. RJPBCS, 7(1): 1756-1759.

Mara, A.R., Pereira, C.A., Gales, A.C., Menezes, L.C., et al. 2006. Bloodstream infections with metallo-beta-lactamaseproducing Pseudomonas aeruginosa: epidemiology, microbiology and clinical outcomes. Antimicrob. Agents Chemother., 50: 388-90.
Nordman, P., Nass, T., Poirel, L. 2011. Global spread of carbapenemase- producing Enterobacteriacae. Emerg. Infect. Dis., 17: 1791-1798.

Queenan, A.M., Bush, K. 2007. Carbapenemases: the versatile betalactamases. Clin. Microbiol. Rev., 20: 440-58.

Rouchelle Tellis, Muralidharan, S., Anand Ignatius Peter. 2013. Evaluation of three phenotypic methods for the detection of metallo-beta-lactamase production in non fermenting gram negative bacilli. Int. J. Biomed. Adv. Res., 04(5): 299305.

Sanjeev Kumar, S.K., Mehra. 2015. Performance of Modified Hodge test and Combined Disc test for Detection of Carbapenemases in Clinical Isolates of Enterobacteriacae. Int. J. Curr. Microbiol. App. Sci., 4(5): 255-261.

Sowmya, G., Shivappa, Ranjitha Shankaregowda, Raghavendra Rao, M., et al. 2015. Detection of Metallo-beta lactamase production in clinical isolates of Nonfermentative Gram negative bacilli. IOSR J. Dent. Med. Sci., 14(10): 43-48.

Walsh, T.R., Toleman, M.A., Poirel, L., Nordman, P. 2005. Metallo- $\beta$ lactamase: the quiet before strom. Clin. Microbiol. Rev., 18: 306-325.

Zavascki, A.P., Barth, A.L., Gaspareto, P.B., et al. 2006. Risk factors for nosocomial infections due to Pseudomonas aeruginosa producing metallo-betalactamase in two tertiary-care teaching hospitals. J. Antimicrob. Chemother., 58: 882-885.

\section{How to cite this article:}

Jayalakshmi, S., and Sathya Pandurangan. 2016. Performance of Modified Hodge Test for Detection of Carbapenemase Producing Clinical Isolates of Pseudomonas aeruginosa. Int.J.Curr.Microbiol.App.Sci. 5(4): 127-132. doi: http://dx.doi.org/10.20546/ijcmas.2016.504.017 